\title{
D1 receptor subtype mediates acute stress-induced dendritic growth of excitatory neurons and gene expression changes associated with stress resilience in the medial prefrontal cortex
}

\author{
Masayuki Taniguchi ${ }^{1}$, Ryota Shinohara ${ }^{1}$, Aliza T Ehrlich ${ }^{2}$, Kentarou Yokogawa $^{2}$, Yuichi Deguchi ${ }^{2}$, \\ Atsubumi Ogawa ${ }^{2}$, Shiho Kitaoka ${ }^{1}$, Akira Sawa $^{3}$, Shuh Narumiya ${ }^{2}$, Tomoyuki Furuyashiki ${ }^{1}$ \\ ${ }^{I}$ Division of Pharmacology, Kobe University Graduate School of Medicine, Japan, ${ }^{2}$ Medical Innovation Center, Kyoto \\ University Graduate School of Medicine, Japan, ${ }^{3}$ Department of Psychiatry, Johns Hopkins University School of \\ Medicine, USA
}

Stress is caused by adverse social environment, and often causes emotional changes including depression and elevated anxiety. Using social defeat stress in mice, we previously reported that social defeat stress preferentially activates the dopaminergic pathway projecting to medial prefrontal cortex $(\mathrm{mPFC})$, and that repetition of social defeat stress attenuates this activation, leading to social avoidance. However, the mechanisms of dopamine action in the mPFC in regulating the resilience to social defeat stress remain elusive. Here we show a role of dopamine D1 receptor in $\mathrm{mPFC}$ excitatory neurons for the stress resilience. Repeated social defeat stress selectively reduced mRNA expression of D1 receptor in $\mathrm{mPFC}$ of susceptible mice. Knockdown of D1 receptor in whole neuronal population or excitatory neurons in $\mathrm{mPFC}$ by viral delivery of artificial microRNA facilitated induction of social avoidance by social defeat stress. Single social defeat stress induced D1 receptor-mediated ERK phosphorylation and c-Fos expression in mPFC neurons. Single social defeat stress increased arborization and spine density of apical dendrites of mPFC superficial layer pyramidal neurons in a D1 receptor-dependent manner. Furthermore, transcriptome analysis showed that single social defeat stress alters expression of multiple genes through $\mathrm{D} 1$ receptor in $\mathrm{mPFC}$, some of which are involved in regulation of neuronal morphology. These results suggest that D1 receptor mediates acute stress-induced dendritic growth of excitatory neurons and gene expression changes associated with stress resilience in $\mathrm{mPFC}$. 